\title{
4th Global CRO Council for Bioanalysis: coadministered drugs stability, EMA/US FDA Guidelines, 483s and Carryover
}

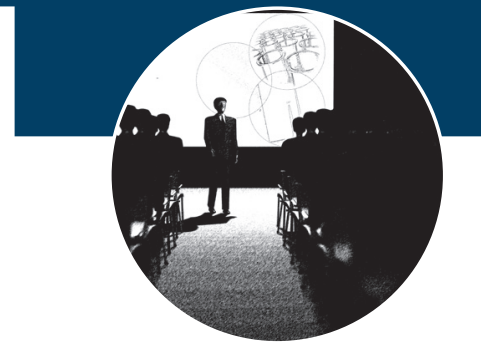

\author{
4th Global CRO Council for Bioanalysis Closed Forum \\ Washington, DC, USA, 23 October 2011
}

The Global CRO Council for Bioanalysis (GCC) was formed in September 2010. Since then, the representatives of the member companies come together periodically to openly discuss bioanalysis and the regulatory challenges unique to the outsourcing industry. The 4th GCC Closed Forum brought together experts from bioanalytical CROs to share and discuss recent issues in regulated bioanalysis, such as the impact of coadministered drugs on stability, some differences between European Medicines Agency and US FDA bioanalytical guidance documents and lessons learned following recent Untitled Letters. Recent 483s and agency findings, as well as issues on method carryover, were also part of the topics discussed.

The 4th GCC Closed Forum was held on 23 October, 2011 in Washington, DC, immediately before the American Association of Pharmaceutical Scientists annual meeting. In attendance were 37 senior-level representatives from $31 \mathrm{CRO}$ s on behalf of six countries. The meeting was chaired by Steve Lowes, who began with the official admonition statement [1-3]. Lowes continued by re-iterating that the goal of GCC is to discuss issues that are particularly relevant to bioanalytical CROs and to communicate the point of view of its members to regulatory agencies, pharmaceutical companies and other bioanalytical organizations.

The question of the frequency of holding the Closed Forum meetings was put to the attending members. From conception, the intent of these meetings is to tie them to major bioanalytical science conferences in order to maximize broadbased attendance and minimize additional travel costs. Attendees agreed that a meeting should be held at least once a year, but additional meeting(s) throughout the year could also be scheduled if a special topic requires discussion.

This meeting's agenda was derived from the results of a survey sent to member companies containing possible 'hot topics' for discussion. The five topics with the most votes were added to the agenda. The subjects discussed included:

- A follow-up on the plan on coadministered stability data collection;

- A review of the new European Medicines Agency (EMA) Guideline on bioanalytical method validation (BMV);
- Lessons learned following a recent US FDA Untitled Letter;

- Recent 483s;

- Method carryover.

GCC action plan regarding stability of coadministered \& coformulated drugs

Starting in late 2009/early 2010, regulatory agencies, and subsequently sponsors, began requesting analyte in matrix stability data that addressed the presence of any coadministered drugs. Several laboratories received FDA Form 483s pertaining to the deficiency of not performing these stability experiments. Due to increased focus by agencies on this topic, the issue was first discussed at the 4th Workshop on Recent Issues in Bioanalysis in 2010 [4] and then more extensively at the 5th Workshop on Recent Issues in Bioanalysis in 2011 [5]. After significant debate at these meetings, it was acknowledged that the potential for one compound to impact the stability of another in biological samples had not been satisfactorily investigated and reported in order to address the regulatory concern. Therefore, following this second discussion, the GCC was approached by prominent scientists from the pharmaceutical industry with a proposal for the GCC to survey its membership regarding the voluntary provision of stability data from coadministered drug stability assessments already underway or completed.

The results from the preliminary survey were published in a White Paper of GCC

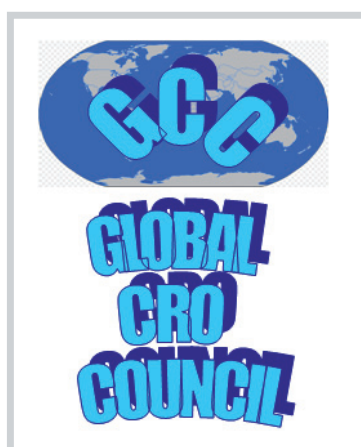

Steve Lowes', Jim Jersey², Ronald Shoup ${ }^{3}$, Fabio Garofolo ${ }^{4}$, Shane Needham ${ }^{5}$, Philippe Couerbe $^{6}$, Tim Lansing 7 , Masood Bhatti ${ }^{8}$, Curtis Sheldon', Roger Hayes ${ }^{10}$, Rafiq Islam" ", Zhongping (John) Lin'2, Wei Garofolo*13, Marc Moussallie $^{14}$, Leonardo de Souza Teixeira ${ }^{15}$, Thais Rocha $^{15}$, Paula Jardieu ${ }^{16}$, James Truog ${ }^{17}$, Jenny Lin ${ }^{17}$, Richard Lundberg ${ }^{18}$, Alan Breau $^{19}$, Carmen Dilger ${ }^{20}$

Authors continued overleaf. 
investigation may be required. A second unjustified occurrence of this calls for a revision to the method prior to continuing.

The EMA guideline has added an assessment of matrix effect to be performed for hemolyzed and lipemic plasma, which, despite being the current industry standard, does not appear in the FDA guidance on BMV. Additionally, the EMA allows for bracketing stability evaluations to be performed for small-molecule studies (stabilities at -20 and $-80^{\circ} \mathrm{C}$ covers samples stored at $-50^{\circ} \mathrm{C}$ ), whereas the Crystal City III White Paper [9] states that there is no consensus on this issue. A last point regarding method validation is that the EMA references to multianalyte (and specifically bioequivalence) studies, where stability in matrix should be demonstrated in the presence of all the analytes. If this is interpreted as addressing coadministered drugs then the above discussion on this topic should be considered.

Routine sample analysis requirements are discussed in both documents and are essentially identical. However, the EMA document requires that if the precision or accuracy of all acceptable runs exceeds $15 \%$, then an investigation should be conducted to determine the root cause and impact on the study data. It is also specified that bioequivalence data may be rejected if this should happen.

Following the presentation, there was a discussion regarding how the members are approaching the need to comply with two different guidance documents. In general, it was recommended to take the more stringent guideline and apply it to their procedures. Members were also counseled to consider the guidance provided by ANVISA [10].

In an attempt to help the member companies interpret the new EMA Guideline, GCC is publishing a White Paper with specific recommendations [11].

\section{Lessons learned following recent Untitled Letters}

An Untitled Letter issued by the FDA in July 2011 regarding the use of 'preparation runs' as well as issues of contemporaneous and accurate documentation, has resulted in an industry-wide re-evaluation of best practices in bioanalysis. Roger Hayes (Cetero Research, TX, USA) presented results from a survey that was conducted within the GCC to get an assessment of the membership's practices regarding 'preparation runs' and documentation. Highlights from the survey were as follows:
- A MS detector requires significant set up and equilibration prior to injecting study samples, in order to ensure accurate and precise results. Each method has its individual needs in this respect, regarding, for example, the number and types of samples that need to be injected to equilibrate the system (it should be noted that the issues were not related to system suitability, but only to system equilibration);

- Half the survey respondents had a standard operating procedure that described the procedure for equilibration as well as acceptance criteria;

- Approximately a quarter of respondents did not have any criteria for system equilibration;

- Most saved the data of system equilibration within the same electronic folder as study data;

- Approximately two-thirds use independently prepared samples for this; however, some do use standards and/or quality control (QC) samples from the current or previously injected batch and even study samples, in some cases;

- Approximately three-quarters of respondents use individual injection vials instead of multi-well plates.

Based upon the survey results, Hayes went on to make some specific recommendations based upon recent reviews and discussion with regulatory authorities. Hayes recommended that consideration should be given when selecting samples to use for equilibration. If standards, QC or study samples are used, there should not be any potential of predetermining the outcome of the analytical run. It is important that instrument equilibration remains independent of the analytical run in order to fully preserve integrity of the bioanalytical data. Appropriate substitutes for standards, QC and study samples for this purpose include neat samples at similar concentrations or pooled samples to create a unique equilibration reagent (including documentation as to the origins of the reagent). In this way, it is impossible to substitute the samples into the actual analytical run and thus avoid the possibility of having two sets of data available that can influence bioanalytical decisions. Furthermore, in order to avoid the possibility of substituting samples in a run, well plates are preferable because individual samples cannot be manipulated as with vials. Identification of equilibration samples should be transparent and stored with the study data. It was recommended to have the 
QC or quality assurance group inspect the project audit trails, which generally provide confirmation of the location of the data, as well as any discrepancies in the injection times of the samples.

On the topic of documentation, basic GLPs state that all data should be recorded directly, promptly and legibly and any original data shall not be obscured in case of a change. Therefore, survey questions were asked in order to determine how GCC members ensured that their data met these criteria. More than three-quarters of respondents used controlled documents to capture the data by using special colored paper, a unique identifying number and/or a time and date stamp.

Other suggestions on how to prevent documentation fraud included the use of electronic laboratory notebooks, only releasing paperwork required for that day's work and use of biometric scanners at key points of the process (e.g., removal and return of study samples from storage). All agreed that further focus on tools and practices to ensure fraud prevention can be anticipated in light of the recent events discussed.

\section{Discussion of recent 483 s \& agency findings}

Robert Nicholson led the discussion on recent Form 483 s or agency findings that had been issued to various GCC member companies. Six different findings were addressed. These are discussed below.

\section{- Use of freshly prepared calibration} standards when determining precision \& accuracy of the method during validation First, the group discussed the use of freshly prepared calibration standards when determining precision and accuracy of the method during validation. Despite the fact that guidance documents and associated White Papers [7-9] state that using qualified bulk preparations is adequate, the inspector indicated that the current expectation was that calibration standards were freshly prepared for each precision and accuracy batch (QC samples could continue to be prepared in bulk for this evaluation). The issue at hand was not stability (in fact, stability data in this case do exist); the concern appears to have been centered around method ruggedness. The members present generally agreed that, during validation, there was logic to proving that the method was sufficiently rugged to support multiple preparations of discrete sets of calibration standards.
- Precision \& accuracy data from separate preparations of QC samples

Related to the preparation of calibration standards, a company was asked during an audit to present precision and accuracy data from separate preparations of QC samples. It is unclear at this time what the reason was for the request; however, current guidances do not require these data. If requested, one source of these data may be available if separate preparations of QC samples occurred during stability evaluations.

\section{- Number of stock solutions used to prepare standard \& QC samples}

This topic pertained to the number of stock solutions used to prepare standard and QC samples. The guidance documents available state that the use of one stock solution to prepare both standards and QC samples is an acceptable practice as long as stability and accuracy have been demonstrated [7-9]. However, there have been FDA Form 483 deficiencies issued for companies that have done this, despite the fact that the stocks were stable and a 'stock check' procedure had proven that the stocks were accurately prepared.

\section{- Shipping of reference standards}

Inadequate documentation associated with the shipping of reference standards has recently come under regulatory scrutiny. Typically, the lack of documents regarding the impact of shipping temperature or other conditions on the reference material is of concern. The US Pharmacopeia acknowledges that the temperatures provided on their labels and Material Safety Data Sheets are intended for long-term storage of the materials and generally do not apply to shipping conditions [12]. Therefore, it is recommended to be as concerned about the shipping conditions of the reference standards as the industry is about the shipping of study samples. Shipping documents (e.g., shipping slips) should be kept as supporting documentation and the condition of the reference standard on receipt at the bioanalytical laboratory should be recorded. One further suggestion voiced, although costly and likely impractical, is to recertify the compound following shipping.

Need to prove stability at every step of the extraction procedure

Stability of the samples over the course of the extraction is often incorporated into the stability tests performed during method validation. However, a citation stated that it was necessary to prove stability at every step of the extraction 
procedure, in order to demonstrate the limits of the process, taking into account possible extended breaks, longer than normal aliquoting and so forth. It was agreed by all those present that one evaluation that covers stability of the entire procedure was adequate and that the stability at each individual step had no added scientific value.

\section{- Interference testing multiple concentrations}

Finally, a company was issued a Form 483 associated with a multianalyte (two analytes) LCMS method. In the case described, inter-analyte interference testing was performed at a single concentration and was not demonstrated in validation at extremes of concentration variance between the two analytes (e.g., assess the impact of ULOQ concentration of one analyte on the LLOQ of the other). In the case presented, the analytes were 2 amu apart and there had been concern of isotopic interference, but the impact had been assessed during method development and there was no issue observed. Furthermore, the analytes were separated chromatographically and each analyte underwent unique fragmentation following collisional activation. Regardless, the agency wanted the proof to exist as part of the validation study.

\section{Impact of background/carryover/ sensitivity during analysis}

For LC-MS/MS methods, the issues of background noise, sensitivity and carryover are dealt with on a day-to-day basis and the subject was presented by Rand Jenkins (PPD, VA, USA). All guidance documents [7-9] require that the peak response of the LLOQ be greater than five-times the noise. In order to determine the impact of contamination or carryover, the industry has always used criteria of $20 \%$ of the LLOQ and $5 \%$ of the internal standard peak response. This has recently been reinforced with the EMA Guidance for method validation. However, if the LLOQ has a $s / n$ ratio of only 5 , then background noise is in fact $20 \%$ of the LLOQ, thereby any assessment of carryover is impractical. This becomes difficult to deal with when there is variable background noise between subjects (as demonstrated by pre-dose samples) or if there is variable carryover between injections.

The above scenario requires that the impact of carryover be properly assessed. One solution is to inject blank matrix samples after high-concentration standards (ULQ) at the beginning and end of the batch. By setting carryover criteria using these blank samples, an evaluation on the reliability of the data can be made. In order to determine the potential for carryover, a proposal was made for including a carryover test in the system suitability evaluation by injecting, first, a blank matrix after a ULQ (must be $<20 \%$ of LLOQ), and then a LLOQ after a ULQ (and the LLOQ response must be within $20 \%$ of nominal concentration).

Jenkins went on to explain how further evaluation of carryover can be performed on a sample-by-sample basis. Using the matrix blanks at the beginning and end of the batch, a 'carryover factor' can be calculated and applied to each sample of the batch, based on the concentration of the previous sample. If there is a possible bias of $>5 \%$ on an unknown sample, because of carryover from the previous sample, the affected sample should be reassayed.

The member companies present do not necessarily use these approaches and one member who used the carryover factor approach did experience a deficiency. However, in general, using a per sample risk assessment approach as described above appears acceptable. What is important is that potential carryover is assessed for all methods and there is a scientific rationale to support the reliability of the data.

\section{Future perspective}

The GCC will continue to provide recommendations on 'hot topics' in bioanalysis of global interest and expand its membership by coordinating its activities with the regional and international meetings held by the pharmaceutical industry. The next GCC Closed Forum is scheduled to take place at the 6th Workshop on Recent Issues in Bioanalysis in San Antonio, TX, USA in March 2012. Please contact the GCC for the exact date and time of the aforementioned meeting and for all membership information.

\section{Acknowledgements}

The GCC would like to thank N Savoie (GCC) for taking the minutes of the 4th GCC Closed Forum and drafting the first version of this conference report. S Martinez and I Dumont (Algorithme Pharma) for critically reviewing this article and W Garofolo (GCC) for organizing the logistics of the meeting. All the member company representatives who have sent comments and suggestions to complete this report and filled in the numerous surveys used to prepare the discussion of the 4th GCC Closed Forum. The GCC member representatives who facilitated and led the discussion of the chosen topics during the 4th GCC meeting. 


\section{News \& Analysis I Conference Report}

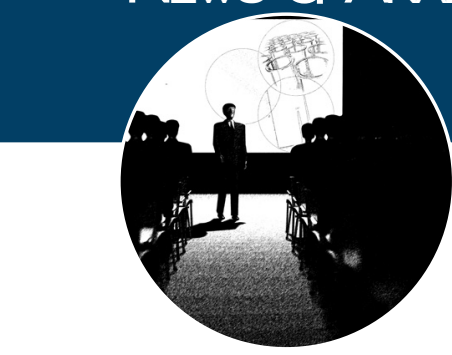

\section{Financial \& competing interests disclosure}

The authors have no relevant affliations or financial involvement with any organization or entity with a financial interest in or financial conflict with the subject matter or materials discussed in the manuscript. This includes employment, consultancies, honoraria, stock ownership or options, expert testimony, grants or patents received or pending, or royalties.

No writing assistance was utilized in the production of this manuscript.

\section{Author affiliations}

Company names in alphabetical order:

'Advion BioServices, Ithaca, NY, USA

${ }^{2}$ Agilux Laboratories, Worcester, MA, USA

${ }^{3}$ AIT Bioscience, Indianapolis, IN, USA

${ }^{4}$ Algorithme Pharma, Laval, Quebec, Canada

${ }^{5}$ Alturas Analytics, Moscow, ID, USA

${ }^{6}$ Atlanbio, Saint-Nazaire, France

${ }^{7}$ BioAgilytix Labs, Durham, NC, USA
${ }^{8}$ BioPharma Services, Toronto, Ontario, Canada ${ }^{9}$ Celerion, Lincoln, NE, USA

${ }^{10}$ Cetero Research, Houston, TX, USA (presently at MPI Research, Mattawan, MI, USA)

"EMD Millipore, St. Charles, MO, USA

${ }^{12}$ Frontage Laboratories, Malvern, PA, USA

${ }^{13} \mathrm{Global}$ CRO Council for Bioanalysis (GCC)

${ }^{14}$ Huntingdon Life Sciences, East Millstone, NJ, USA

${ }^{15}$ ICF, Goiania, Brasil

${ }^{16}$ ICON Development Solutions, Whitesboro, NY, USA

${ }^{17} \mathrm{JCL}$ Bioassay USA, Hoffman Estates, IL, USA

8MEDTOX Laboratories, St Paul, MN, USA

${ }^{19} \mathrm{MPI}$ Research, Mattawan, MI, USA

${ }^{20}$ Nuvisan GmbH, Neu-Ulm, Germany

${ }^{21}$ Pharma Medica, Toronto, Ontario, Canada

${ }^{22}$ PharmaNet Canada, Quebec City, Quebec, Canada

${ }^{23}$ PPD, Richmond, VA, USA

${ }^{24}$ Protech Pharmaservices, Taipei City, Taiwan

${ }^{25}$ Pyxant Labs, CO Springs, CO, USA

${ }^{26}$ Ricerca Biosciences, Concord, OH, USA

${ }^{27}$ Smithers Pharma, Wareham, MA, USA

${ }^{28}$ Tandem Labs, West Trenton, NJ, USA

${ }^{29}$ Tandem Labs, Durham, NC, USA

${ }^{30}$ Warnex Bioanalytical, Laval, Quebec, Canada

${ }^{31}$ WIL Research Company, Skokie, IL, USA

${ }^{32}$ Worldwide Clinical Trials, Austin, TX, USA

${ }^{33}$ XenoBiotic Laboratories, Plainsboro, NJ, USA

\section{References}

1 Premkumar N, Lowes S, Jersey J et al. Formation of a Global Contract Research Organization Council for bioanalysis. Bioanalysis 2(11), 1797-1800 (2010).

Dumont I, Garofolo F. Conference report: the 1st conference in Asia-Pacific on recent issues in bioanalysis. Bioanalysis 3(7), 723-731 (2011).

3 Breda M. Garofolo F, Caturla MC et al. The 3rd Global CRO Council (GCC) for Bioanalyis at the International Reid Bioanalytical Forum. Bioanalysis 3(24), 2721-2727 (2011).

4 Savoie N, Garofolo F, van Amsterdam P et al. 2010 White Paper on recent issues in regulated bioanalysis and global harmonization of bioanalytical guidance. Bioanalysis 2(12), 1945-1960 (2010).
5 Garofolo F, Rocci ML, Dumont I et al. 2011 White Paper on recent issues in bioanalysis and regulatory findings from audits and inspections. Bioanalysis 3(18), 2081-2096 (2011).

6 Lowes S, Jersey J, Shoup R et al. Recommendations on: internal standard criteria, stability, incurred sample reanalysis and recent 483s by the Global CRO Council for bioanalysis. Bioanalysis 3(12), 1323-1332 (2011).

7 European Medicines Agency. Guideline on bioanalytical method validation. European Medcines Agency, Committee for Proprietary Medicinal Products (CPMP), London, UK (2011).

8 US FDA. Guidance for Industry: bioanalytical method validation. US FDA, Center for Drug Evaluation and Research, MD, USA (2001).
9 Viswanathan CT, Bansal S, Booth B et al. Quantitative bioanalytical methods validation and implementation: best practices for chromatographic and ligand binding assays. Workshop/Conference Report. AAPS J. 9(1), E30-E42 (2007).

10 ANVISA. Manual for Good Bioavailability and Bioequivalence Practices. Brazilian Sanitary Surveillance Agency, Brazil (2002).

11 Boterman M, Doig M, Breda M et al. Recommendations on the Interpretation of the new European Medicines Agency Guideline on bioanalytical method validation by Global CRO Council for Bioanalysis (GCC). Bioanalysis (In press).

12 USP. Shipping Conditions for USP Reference Standards. USP, Rockville, MA, USA (2008). 